\title{
Wavelength Dependent Light Tunable Resistive Switching Graphene Oxide Nonvolatile Memory Devices
}

\author{
Ayoub H. Jaafar ${ }^{a, b}$ and N. T. Kemp ${ }^{a, *}$ \\ ${ }^{a}$ Department of Physics and Mathematics, University of Hull, Cottingham Rd, HU6 7RX, Hull, United \\ Kingdom \\ ${ }^{b}$ Physics Department, College of Science, University of Baghdad, Baghdad, Iraq
}

\begin{abstract}
This paper reports on the first optically tunable graphene oxide memristor device. Modulation of resistive switching memory by light opens the route to new optoelectronic devices that can be switched optically and read electronically. Applications include integrated circuits with memory elements switchable by light and optically reconfigurable and tunable synaptic circuits for neuromorphic computing and braininspired, artificial intelligence systems. In this report, planar and vertical structured optical resistive switching memristors based on graphene oxide are reported. The device is switchable by either optical or electronic means, or by a combination of both. In addition the devices exhibit a unique wavelength dependence that produces reversible and irreversible properties depending on whether the irradiation is long or short wavelength light, respectively. For long wavelength light, the reversible photoconductance effect permits short-term dynamic modulation of the resistive switching properties of the light, which has application as short-term memory in neuromorphic computing. In contrast, short wavelength length induces both a reversible photoconductance effect and irreversible changes due to reduction of the graphene oxide. This has important application in the fabrication of neural networks with factory defined weights, enabling the fast replication of artificial intelligent chips with pre-trained information.
\end{abstract}

\section{Introduction}

Leon Chua's pioneering work on the theoretical description of memristors[1] combined with HP Labs link to memristor switching in metal oxides[2], and the more recently demonstrated use of memristors

* Corresponding author. E-mail: N.Kemp@hull.ac.uk (Neil Kemp) Ph: +44 1482466149 
as artificial synapses in neuromorphic computing[3][4] has sparked enormous resurgence in these twoterminal, non-volatile memory devices. Among the emerging memory technologies including ferroelectric RAM, magnetic RAM and phase change RAM, resistive switching RRAM is considered as a promising candidate for next generation memory owing to its low power operation, fast switching speeds, high-density crossbar integration, long data retention and endurance capabilities[5-8]. Memristors are typically switched between two resistance states, a high resistance state (HRS) and a low resistance state (LRS), when an electrical bias is applied. However there are significant advantages if devices can as well be switched by optical means since optical signalling supports higher bandwidth communication, faster transmission speeds and the ability to decouple signals from electronic noise. A suite of potential new applications include; switchable memory elements in optical communications and photonic integrated circuits, optically tunable synaptic elements and new types of light sensors that can be used in cellular neural network cameras[9-11]. The latter having the important advantage that some in-memory processing of the image (e.g. edge detection) can be done directly at the level of the focal plane on the camera chip, which eliminates problems associated with the slow data transfer bottleneck between the optical sensor and the main processor. The fast parallel nature of light patterning also facilitates optical re-programming (or erasure) of memory banks or the ability to fine-tune memristors electronic properties during fabrication to desired quality-control specifications. We believe the optical control of memristors has significant advantages in neuromormphic computing, whereby light acts as an additional degree of freedom to dynamically adjust the synaptic learning properties (e.g. plasticity) of indvidual memory elements or regions of elements[12], thereby providing enhanced learning/training properties and/or new circuit functionality. In many ways, the use of light in this manner affords the ability to control a network in much the same way that bio-chemical reactions mediate changes in the learning and the structure of biological brains during its lifetime.

To date, relatively few optical memristors have been reported. Devices have been demonstrated that exhibit optical reading[13][14], and optical switching[15-17], and recently we reported[12,18,19] on a polarization specific switching device that exhibited both a latched Set and latched Reset (erase) functionality. In this work, we report for the first time a graphene oxide (GO) based-resistive switching device that shows both reversible and irreversible effects with light exposure, as well as standard resistive switching properties under dark conditions. Resistive switching has been demonstrated in GO based devices before[20-28], however, to the best of our knowledge, optical modulation of the resistive switching in GO-based memories has not been shown. Graphene oxide has excellent optical and electronic properties which make it a useful candidate for optoelectronic applications[29-31]. The 
reduction of graphene oxide to reduced graphene via optical means improves the electrical properties of GO films[32,33], whilst also enabling band gap engineering. In this report we utilize both the photocurrent response of GO films[34,35] and optical reduction of the GO to control the electronic properties of our memristor devices.

\section{Methods}

The graphene oxide (GO) thin-films, Figure 1(a), were prepared from commercially available GO flakes (4 mg/ml dispersion in water purchased from Sigma Aldrich). This solution was diluted in deionized water (DI) in different ratios (GO:DI), (1:50, 1:40, 1:30, 1:20, 1:10, and 1:2), as shown in Figure 1b, to give different thicknesses of GO films of (70,105, 130, 200, 370, and 500) nm, respectively. The solutions were deposited onto two different device types, vertical and planar device. The substrate for the vertical devices and planar devices were ITO coated glass substrates (purchased from Delta Technologies) and interdigitated ITO substrates (purchased from Ossila and consisting of 100 micron wide ITO electrodes with spacing of 50 micron), respectively. In both cases thin-films of GOx were made by drop casting. The samples were then annealed at $45^{\circ} \mathrm{C}$ for 30 minutes in air. In the case of the vertical structure devices, $200 \mathrm{~nm}$ thick top silver electrodes were deposited by thermal evaporation under vacuum conditions using a shadow mask containing $400 \mu \mathrm{m}$ diameter circles. The schematic of the two optical memristors types is shown in Figure 1(c) and 1d). I-V sweeps were carried out using a probe station equipped with an HP4140B source-meter unit. Optical illumination of the devices was done by red $(625 \mathrm{~nm})$, green $(525 \mathrm{~nm})$, blue $(465 \mathrm{~nm})$ and UV $(380 \mathrm{~nm})$ LEDs that were adjusted to give an average power per unit area of $44 \mathrm{~mW} / \mathrm{cm}^{2}$.

\section{Results}

Two device architectures consisting of vertical and planar electrode geometries were investigated, as shown by device schematics in Figure 1c) and 1d), respectively. The GO vertical devices consist of a large-area ITO bottom electrode, a middle GO layer and thermally deposited silver top electrodes. The GO planar devices instead consisted of a GO layer drop-casted directly onto a substrate consisting of interdigitated ITO electrodes on glass. The variation in the thickness of the active GO layer, $500 \mathrm{~nm}$ (vertical) vs. $50 \mu \mathrm{m}$ (planar), plays a significant role in their electrical and optical properties. The results are reported separately in the two sections below. 
a)

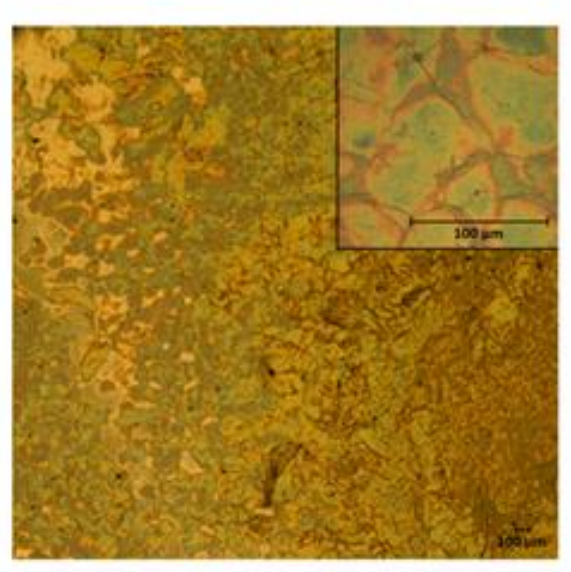

c)

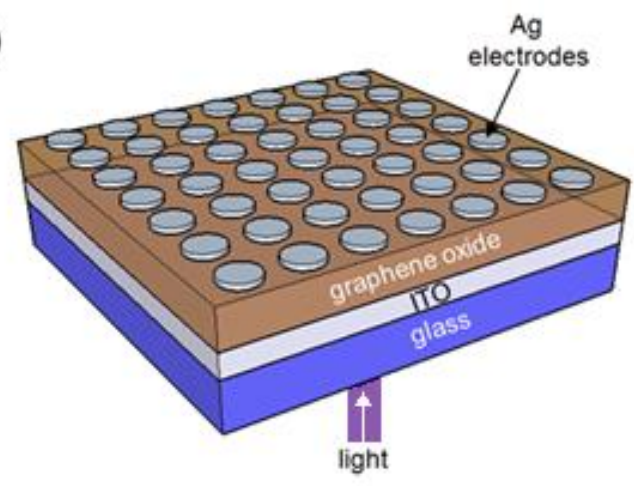

b)

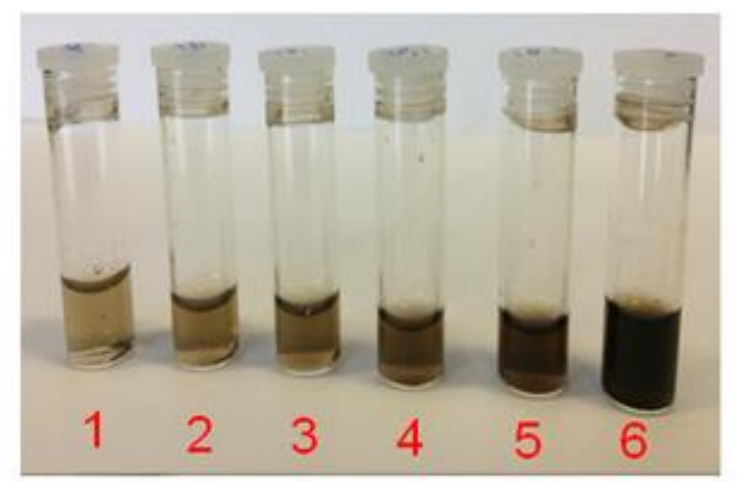

d)

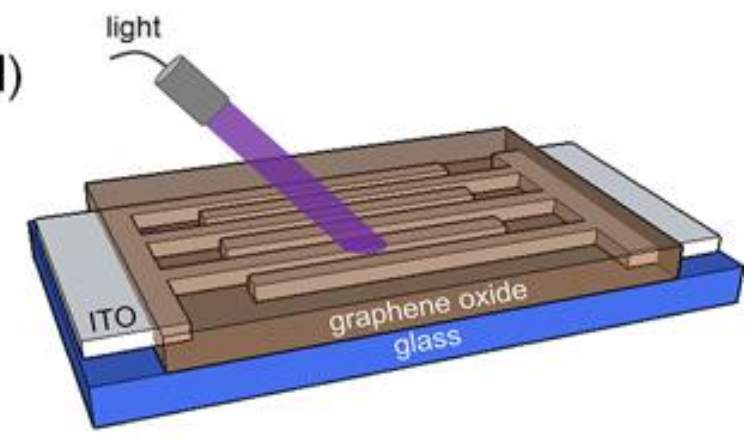

Fig. 1. a) Optical microscopy image of a drop-casted GO film. b) GO solutions with different concentrations of 1:50,1:40, 1:30,1:20, 1:10, and 1:2 for samples 1 to 6 , respectively. c) Schematic of the optical resistive switching device consisting of GO deposited between ITO and Ag electrodes (vertical structure). d) Schematic of the optical resistive switching device consisting of GO deposited between ITO electrodes (planar structure).

\subsection{Planar Devices}

Figure 1(a) shows the optical microscope image of an as-deposited GO film deposited on a glass substrate to form a uniform, thin-film of GO flakes. In contrast, Figure 2(a) shows the optical microscope image of a GO film deposited onto a planar device consisting of interdigitated ITO electrodes. The image shows the device after the application of an electroforming process, which was found necessary (only in the planar devices) to initiate resistive switching. Before the forming process, devices had high resistivity and no resistive switching. However, when a device was swept between $-8 \mathrm{~V}$ and $+8 \mathrm{~V}$ with a compliance current of $10 \mathrm{~mA}$, see inset Figure 2(b), conductive paths across the GO film regions between the ITO electrodes were formed, most likely due to reduction of the GO via a Joule heating process to form partially reduced form of GO, as detailed in the discussion section. After this process, it was found the 
GO layer between the ITO electrodes contained bubble-like structures (mostly likely due to gas evolution), as shown in Figure 2(a), and the device acted as a memristor with the characteristic signature of a pinched hysteresis loop through the origin, as can be clearly seen by the I-V loops shown in Figure 2(b). The observed bipolar resistive switching was found to be reproducible and the switching SET and RESET voltage occurred at much lower voltage, between $\pm 2 \mathrm{~V}$, than that used in the electroforming step.
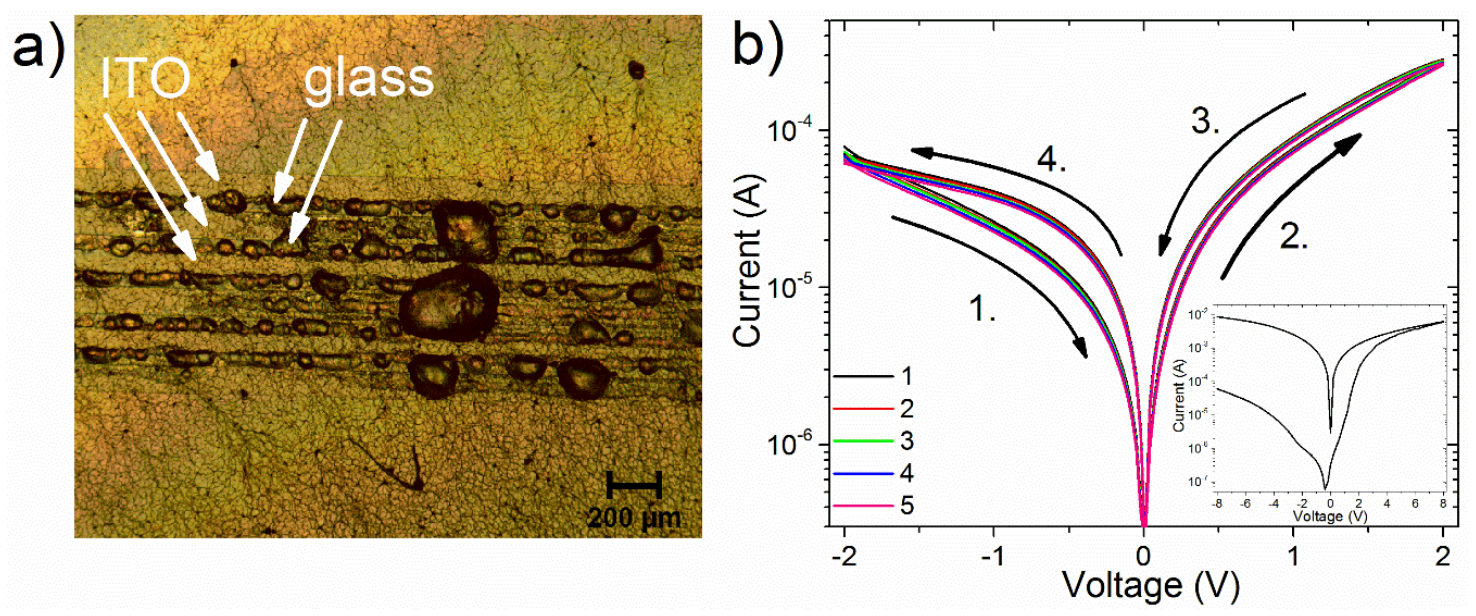

Fig. 2. a) Optical microscope image of a planar GO device after an initial forming step to convert the device into a memristor device. The dark regions between the ITO electrodes is bubbling of the GO film due to reduction of the GO by Joule heating. b) I-V characteristics of the memory device after the forming step. The inset shows the I-V properties during the forming step.

Once the planar memristor device was formed by electroforming, the effect of optical illumination of the device on the electronic switching was investigated. Figure 3(a) shows the photocurrent response of the planar device to UV light $(\lambda \approx 380 \mathrm{~nm})$ as a function of time at a constant voltage bias of $0.1 \mathrm{~V}$ and with the illumination cycled between $\mathrm{ON}(60 \mathrm{~s})$ and $\mathrm{OFF}(60 \mathrm{~s})$. The inset also shows the photocurrent response with illumination by red $(625 \mathrm{~nm})$, green $(525 \mathrm{~nm})$ and blue $(465 \mathrm{~nm})$. Illumination by all four wavelength types produces a photo-response, whereby the current increases and decreases when the light source is $\mathrm{ON}$ and $\mathrm{OFF}$, respectively. This is also leads to changes in the resistive switching properties of the device as shown by the curent-voltage sweeps, performed on the device before and after light exposure, as shown in Figure 3(b). 
Significant differences in the response of the device with illumination by UV and visible light (RGB) are clearly shown. UV illumination produces more than 10 times the change in the current than illumination by visible light. Also, whereas illumination by RGB light is perfectly reversible, as indicated by the flat baseline with repeated on/off cycles and the current of the device returning to its pre-illuminated value at the end of an off cycle, illumination by UV includes both a reverisble and an irreversible component. With repeated ON/OFF cycling the baseline value increases with time indicating that in addition to the reversible optical effect there is a significant irreversible effect present. Figure 3(c) examines this more closely by illuminating the device to UV light for 33 minutes and then leaving the device in the dark for an extended amount of time, approximately 1 hour. In this case, the irreversible change in the conductance of the device can be clearly seen. The effect of this on the memristance properties of the device is also evident, as shown by the current-voltage plots in the inset. We ascribe this irreversible effect to partial reduction of the graphene oxide, as will be detailed in the discussion section.
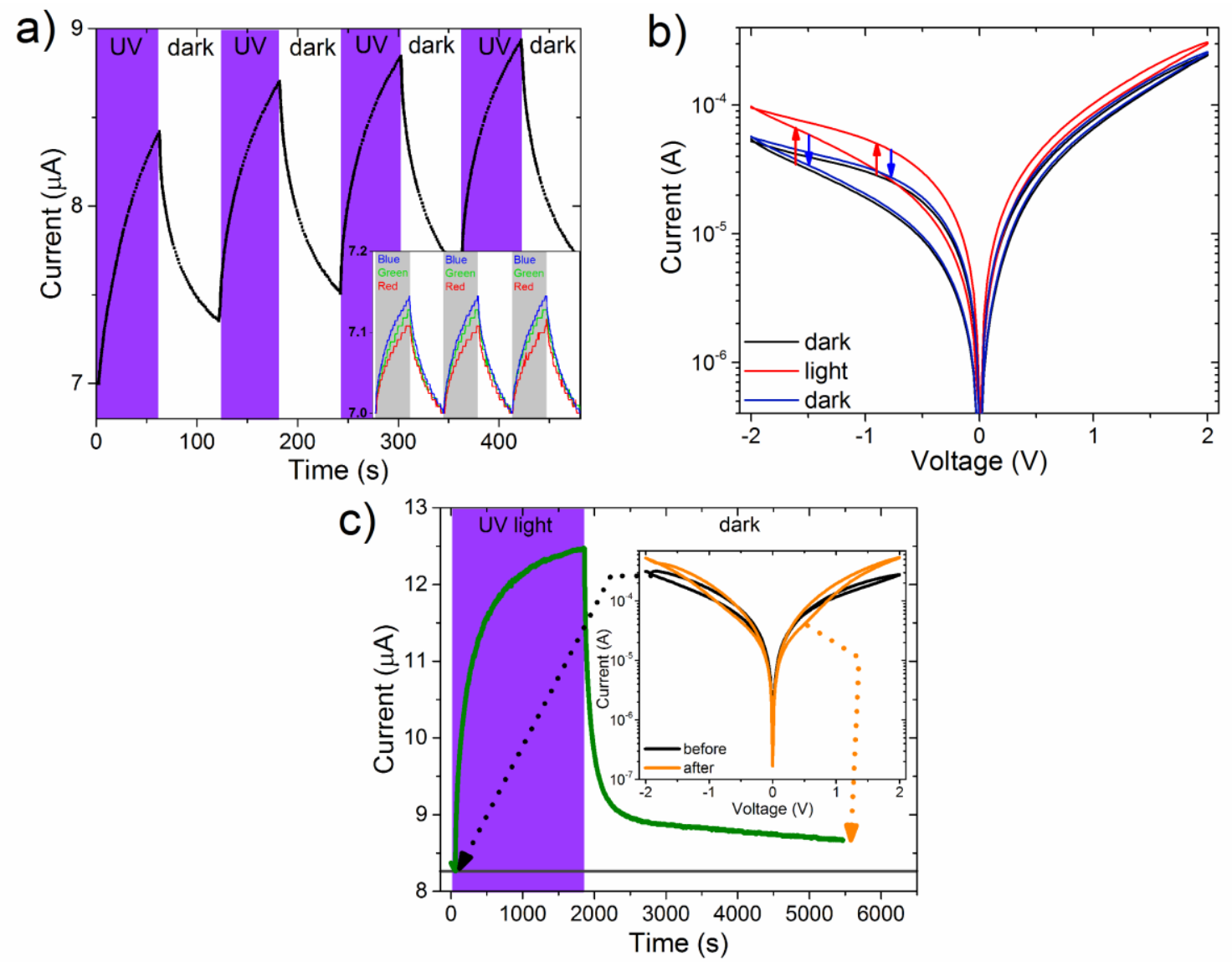

Fig. 3. a) Dark and light photocurrent response of a planar device (ITO/GO/ITO) at $0.1 \mathrm{~V}$ when irradiated with a $380 \mathrm{~nm}$ UV light source as a function of time and showing both a reversible and irreversible components. The inset shows the response of the device to red $(625 \mathrm{~nm})$, green $(525 \mathrm{~nm})$ and blue (465 
$\mathrm{nm}$ ) light (using the same irradiance). In this case, the longer wavelength light produces only a very small photoreponse effect, which is perfectly reversible. b) Current-Voltage properties of the same device that is initially in the dark (black curve), after irradiation with UV light (red curve), and a short period in the dark (blue curve). c) Photocurrent repsonse of the device to UV light during a long exposure time (33 minutes) followed by measurement in the dark for 1 hour and showing an irreversible change in the conductance. The inset show the current-voltage properties before (black) and after (orange) the photocurrent measurements.

\subsection{Vertical Devices}

As was found in the planar structured GO memristor device, the vertical structured GO memristors similarly exhibited a photoresponse and changes in the memristor switching properties upon irradiation with UV light. In contrast to the planar devices, it was found that an initial electroforming step was not needed in the vertical structured devices. Figure 4(a) illustrates the photocurrent response of an ITO/GO/Ag device at a constant voltage of $0.1 \mathrm{~V}$ as a function of time. Upon exposure to $380 \mathrm{~nm}$ light the current increased from about $4.3 \times 10^{-8} \mathrm{~A}$ to about $1 \times 10^{-7} \mathrm{~A}$ during the first 5 minutes. After turning OFF the UV light, the photocurrent decreased to about $6.3 \times 10^{-8} \mathrm{~A}$ after a period of 3 minutes. With subsequent ON/OFF cycling the baseline value increased with time indicating that in addition to the reversible optical effect there is a significant irreversible effect present, as was similarly observed in the case of the planar devices.

The influence of UV light illumination on the memristor switching properties is shown in Figure 4 (b). The graph shows the I-V curves of the vertical structured optical memristor in dark conditions and with illumination with UV light. The illumination shifts the I-V curves of both the ON and OFF state currents to higher values (red curve) in comparison with the initial I-V sweep (black curve) that was measured in the dark. Turning the light OFF, and waiting for a period $150 \mathrm{~s}$, demonstrates the effect is partially reversible, as shown by the return the I-V (blue) curve close to its initial position. 

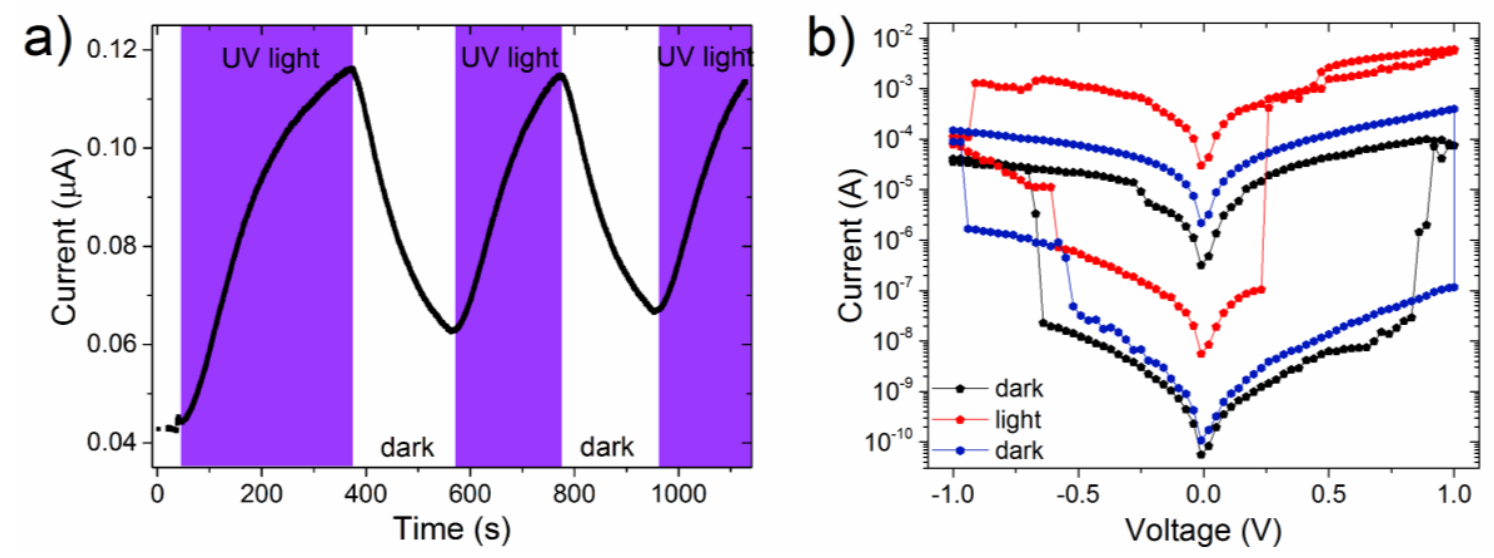

Fig. 4. a) Dark and light photocurrent response of vertical device (ITO/GO/Ag) at $0.1 \mathrm{~V}$ when irradiated with a $380 \mathrm{~nm}$ UV light source as a function of time. b) Optical modulation of the electronic switching characteristics of an ITO/GO/Ag memristor (370 nm thick of GO) in dark (black curve), during irradiation with UV light (red curve) and in dark again (blue curve).

The electronic switching properties of the vertical devices were further studied by examining the currentvoltage switching properties of devices with different GO film thickness, as shown in Figure 5 (a). The applied voltage was swept between $-1 \mathrm{~V}$ and $+1 \mathrm{~V}$ with a current compliance of $10 \mathrm{~mA}$ to protect the devices from breakdown. All devices showed bipolar resistive switching properties and unlike the planar structured devices, none of the devices required a forming step to initiate switching. However, there were significant differences in the resistive switching properties as a function of the GO film thickness. Devices having thin GO films exhibited abrupt transitions between the HRS and LRS states whilst devices with thick GO layers had smooth current - voltage curves. The ON and OFF currents and the separation between $\mathrm{ON}$ and OFF resistance states were also found to be dependent on the GO film thickness. Increasing the film thickness from $70 \mathrm{~nm}$ to $370 \mathrm{~nm}$ decreased both the ON and OFF currents, whilst interestingly, the conductance ON/OFF ratio increased from 1.4 to 10,000, as shown in Figure 5 (b). A further increase in the GO film thickness to $500 \mathrm{~nm}$, decreased both the ON and OFF currents and diminished the conductance $\mathrm{ON} / \mathrm{OFF}$ ratio to a value of $\approx 5$. It is apparent from the graph that an optimum value of the conductance ON/OFF ratio exists for a specific film thickness. [Note: in all cases the conductance $\mathrm{ON} / \mathrm{OFF}$ values were measured at $0.14 \mathrm{~V}]$ 

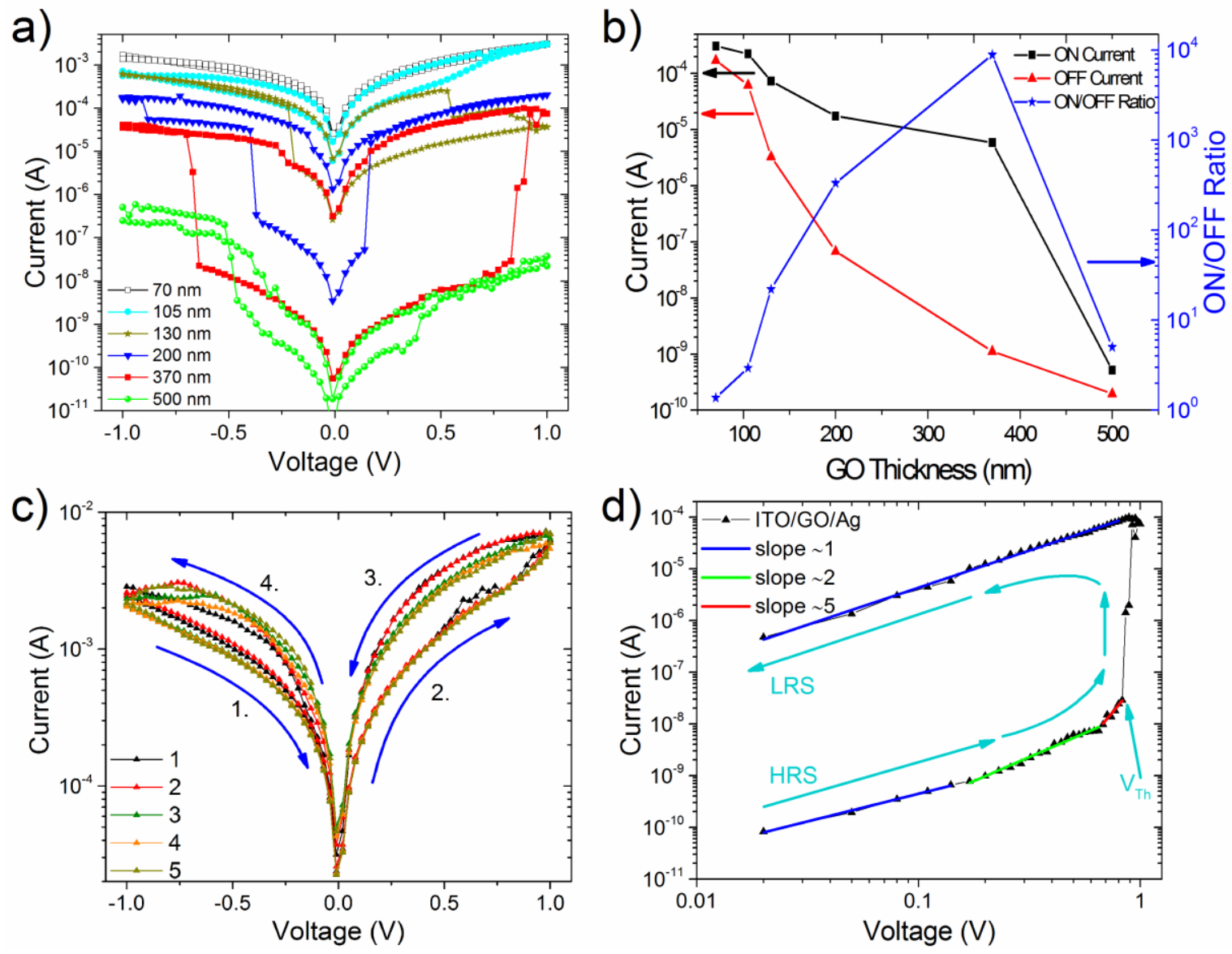

Fig.5. a) I-V characteristics of vertical structured ITO/GO/Ag optical memory devices with different GO film thicknesses. b) The conductance ON/OFF ratio and the ON and OFF currents as a function of GO film thickness at $0.14 \mathrm{~V}$. c) Five successive I-V sweeps of an ITO/GO/Ag device (d 105 nm) showing the reproducibility of device performance. d) Curve fitting using an SCLC model on an ITO/GO/Ag optical memristor with a $370 \mathrm{~nm}$ thick of GO film.

Reproducibility of the electronic memristor switching of the memristor device is demonstrated in Figure 5(c). The graph shows five successive I-V sweeps of an ITO/GO/Ag device with a GO film thickness of $105 \mathrm{~nm}$. It is apparent from this graph that the device exhibited good reliability, and no degradation was observed after 5 successive I-V sweeps.

In Figure 5(d) the conduction mechanism of the GO memristor device is examined by plotting the positive part of the I-V curve on a $\log$ - log scale. Initially the device was in the HRS (OFF) state from 0 to $0.83 \mathrm{~V}$. Within this range the device exhibits linear behaviour with a slope of $\sim 1$ from 0 to $0.14 \mathrm{~V}$. 
This can be attributed to Ohmic conduction, whereby the charge transport arises from thermally generated carriers. At higher applied bias, $0.17 \leq \mathrm{V} \leq 0.65$, the slope changes to $\sim 2$, and the current exhibits the voltage square dependence, $I \propto \mathrm{V}^{2}$, which can be attributed to the trap-controlled space charge limited current (TC-SCLC) described by the Mott-Gurney law,[36] and given by:

$$
J=\frac{9 \varepsilon \mu V^{2}}{8 d^{3}}
$$

where $J$ is the current density, $\varepsilon$ is the dielectric constant, $\mu$ is the free carrier mobility, $V$ is the applied voltage, and $d$ is the insulator thickness.

In the region of $0.68 \leq \mathrm{V} \leq 0.82$ a much steeper rate of current increase occurs with a slope of about 5 , indicating that all traps are filled. The conduction in this region can be ascribed to trap-filled space charge limited conduction (TF-SCLC). At the threshold of $0.83 \mathrm{~V}$, an abrupt transition in the current takes place, switching the device from the HRS (OFF) to the LRS (ON) state. In the case of the LRS, the conduction mechanism is completely dominated by Ohmic conduction for the return voltage region from $1 \mathrm{~V}$ to $0 \mathrm{~V}$. This region shows a linear dependence of current with applied voltage, I $\propto \mathrm{V}$, with a slope of $\sim 1$. The fitting graph suggests that the conduction mechanism corresponding to the electrical bistability in our optical device is attributed to the Space-Charge-Limited-Current (SCLC).

\section{Discussion}

Graphene oxide (GO) is chemically the same as graphite oxide but is structurally very different as it consists of exfoliated monolayers or few-layered stacks. The stacked structure of GO contains many oxide functionalities typically epoxy, hydroxyl, carbonyl and carboxyl groups on a carbon framework. It can be viewed as graphene with the oxygen functional groups decorating the basal plane and edges. Since the $\mathrm{sp}^{2}$ interplanar spacing is disrupted the material acts as an insulator with a typical band gap of 4.2 $5.5 \mathrm{eV}$. The conductivity of GO is ionic in nature and is highly dependent on its environment. It is known to vary between $5 \times 10^{-6} \mathrm{~S} / \mathrm{cm}$ and $4 \times 10^{-3} \mathrm{~S} / \mathrm{cm}$, depending on its local environment[37][38]. Removal of the oxygen species to form reduced graphene oxide (r-GO) allows re-establishment of the graphene like carbon lattice network, hybridised bonding and formation of a delocalised network of charge carrying electrons[39], which greatly increases the conductivity of the material to approximately 3 order of magnitude less than pristine graphene. Since removal of the oxide functionalities also modifies the 
band gap[40][41], this provides a control mechanism for band-gap engineering, which opens the route to many potential optical and photovoltaic applications.

The GO-based non-volatile memory devices with planar and vertical structures both show reliable and repeatable resistive switching properties at low applied voltages. In the case of planar devices, a forming process was needed to initiate resistive switching. The mechanism of forming in GO resistive switching devices has not been definitively established. In the case of metal-oxide systems it is expected to be due to the movement of defects in the material itself (e.g. oxygen vacancies) or the migration of metal ions from the electrodes. We expect a similar mechanism occurs in GO and there is increasing evidence to support that this is mediated by oxygen vacancy migration.[28] However, it is unclear why a forming step is required in planar devices but not vertical devices, which warrants further studies. Certainly, in vertical devices the migration of metal ions and oxygen would be more difficult since these atoms cannot move through the graphene plane, but must circumvent around the edges of flakes (note: graphene layers are impermeable[42] to all atoms and molecules, even hydrogen, which explains the bubbling effect observed in films, since any gas that arises from the electroforming step cannot diffuse easily out of the graphene layers and instead forms a bubble, as shown in Figure 2.) However, in vertical devices, since only one pathway is needed to form a conducting channel, the chance of this occuring naturally (i.e. without the need of a forming step) across the much thinner film thickness (100-200 nm) would be significantly higher than that of the planar device, which has a much larger electrode separation $(50 \mu \mathrm{m})$. In the case of the vertical devices the naturally formed conductive channel may be a very small localized region where many vertically stacked flakes have a similarly positioned edge.

The highly anisotropic nature of the graphene flakes would also be expected to play a role in the device's electronic properties and forming process since the current paths would be very different in the vertical and planar devices. For vertical devices, since the graphene flakes tend to lie parallel to the surface, the current path will be in the direction perpendicular to the plane of the graphene flakes, whereas in planar devices, the current path will be along the graphene flakes. In addition, the heterogeneous nature of conduction in the films, due to disorder, also needs to be considered since reduced GO is said to contain regions of delocalized states with relatively high conductance that are separated by disordered regions with localized states, where the conduction is by hopping[43].

The planar and vertical GO devices exhibit significant differences in the current-voltage curves and switching properties, as shown by Figures 2 b) and 4 b). Planar devices exhibit resistive switching with 
smooth transitions between the HRS and LRS. This type of switching is generally consistent with nonfilamentary like behaviour. In contrast, vertical devices exhibit abrupt switching characteristics and evidence of multilevel states, which is typically associated with either filamenary-like behaviour or a space charge mechanism. In addition, the conductance ON/OFF ratio of vertical devices is much larger, reaching as high as $10^{4}$.

Previous studies have attempted to clarify the origin of resistive switching in GO devices. In a crossbar device structure[44] consisting of GO thin film sandwiched between top and bottom aluminium electrodes, resistive switching behaviour was observed and attributed to the formation and rupture of conductive paths that are induced by the back and forth movement of oxygen ions in the film and the formation of barrier layer $\left(\mathrm{Al}_{2} \mathrm{O}_{3}\right)$ at the interface of $\mathrm{GO} / \mathrm{Al}$ top contact during the SET and RESET processes. It was proposed that deposition of the top $\mathrm{Al}$ contact on the GO film formed an insulating interface layer through a redox reaction between Al and GO films. Application of high electric field diffuses the oxygen ions out of the locally concentrated electric field regions and produces highly reduced GO paths within the GO film. The electric field also diffuses the metal ions into the insulating interface layer and that forms Al conductive channels. Consequently, conductive channels occur across both the GO film and interface layer that switch the device to the LRS. Reversing the polarity of the applied bias diffuses the oxygen ions back into the conductive channel regions of GO film and that switches the device to the HRS state[28,45]. However, in contrast to this previous work, our devices were not made with aluminium, but instead either had both ITO as electrodes (planar) or ITO and Ag as electrode (vertical). Certainly, it is known that $\mathrm{Ag}$ atoms are relatively mobile and can form metallic atomic-sized bridges with voltage pulses [46] but it is less clear if this occurs in the case of ITO. A further complication is whether these bridges could form in a vertical device due to the impermeable nature of GO.

The main focus of this work is the optical tunable properties of the memristor device. Exposure of the planar and vertical structured memristor devices to light produced significant changes in the electrical and switching properties of the devices. Both devices types exhibited a sizeable photoconductance effect. This effect is well-known in graphene oxide and is attributed to photoexcitation of electrons into the conduction GO conduction band. The band gap of GO is expected to be $4.2-5.5 \mathrm{eV}$, which is consistent with the photoreponse of the devices, which were 10 times larger in the case of short wavelength UV light $(380 \mathrm{~nm}$ or $3.3 \mathrm{eV})$ in contrast to red wavelength light $(625 \mathrm{~nm}$ or $2.0 \mathrm{eV})$. However, GO can also be reduced to a more conductive form through a complex light induced heating effect which depends not only on the wavelength of the light but also its intensity.[47][48] In this work we observed that in the 
case of low intensity light at long wavelength, the photoconductance effect is perfectly reversible, as seen by others in the wavelength range from $455 \mathrm{~nm}$ to $980 \mathrm{~nm}$ [49]. However, in the case of shorter wavelength light, the photoconductance effect in the memristor devices was only partially reversible and this can be attributed to photoinduced reduction of the graphene oxide. This occurred in both planar and vertical device structures and significantly altered the memristor switching properties of the devices, as reported in Figures 3b) and 4b).

Light tunable memristor switching has important application in neuromorphic computing applications since it allows control of the learning properties of artificial synapses by light[12]. The ability of light to pattern either individual or entire regions containing many memristors can be used as an additional degree of freedom to independently control and govern large scale neuromorphic systems, enabling a type of hierarchical management that could be used to modify, fine-tune or completely change the learning abilities of the system when needed. Illumination of the devices by UV light shifted both the ON and OFF states to higher current values whereas turning the light OFF shifted the ON and OFF states to lower (initial) current values. In this work, we attribute the increase of the current upon UV irradiation to the photocurrent response of the GO film[50,51]. Exposure of the device to UV light generates hole and electron pairs and application of an electric field separates these pairs which result in a photocurrent response. Both planar and vertical devices exhibited changes in the shape of the I-V curves during and after exporsure to light. In the case of the vertical devices this was significantly greater and also occurred along with large changes in the threshold switching voltages.

\section{Conclusions}

In summary, this paper reports on the first optically tunable graphene oxide memristor device. Two types of device structures, planar and vertical, are reported. In both cases the devices are switchable by either optical or electronic means, or by a combination of both. Both devices exhibit unique wavelength dependent properties. Reversible and irreversible optical switching properties arise from the use of short wavelength light, whereas for long wavelength light the switching is purely reversible. The reversible optical switching is due to a photoconductance effect, whereas the irreversible switching property is due to reduction of the graphene oxide. The optical modulation of the resistive switching properties using long wavelength light has application in emulating short-term memory effects in neuromorphic 
computing and weight-adjustable learning in neural networks. In contrast, the short wavelength effect, causing irreversible changes, has important application in setting the initial value of weights in neural network to pre-defined values.

\section{Acknowledgements}

We would like to sincerely thank the Iraqi Ministry of Higher Education and Scientific Research (University of Baghdad) for supporting and part funding of this work. The authors would like to acknowledge the contribution of the COST Action IC1401.

\section{References}

[1] L.O. Chua, Memristor - The Missing Circuit Element, IEEE Trans. Circuit Theory. CT-18 (1971) 507-519.

[2] D.B. Strukov, G.S. Snider, D.R. Stewart, R.S. Williams, The missing memristor found., Nature. 453 (2008) 80-3. doi:10.1038/nature06932.

[3] L.O. Chua, Memristor, Hodgkin-Huxley, and edge of chaos., Nanotechnology. 24 (2013) 383001. doi:10.1088/0957-4484/24/38/383001.

[4] O. Šuch, M. Klimo, N.T. Kemp, O. Škvarek, International Journal of Electronics and Communications ( AEÜ ) Passive memristor synaptic circuits with multiple timing dependent plasticity mechanisms, 96 (2018) 252-259. doi:10.1016/j.aeue.2018.09.025.

[5] Y.C. Yang, F. Pan, Q. Liu, M. Liu, F. Zeng, Fully Room-Temperature-Fabricated Nonvolatile Resistive Memory for Ultrafast and High-Density Memory Application, Nano Lett. 9 (2009) $1636-1643$.

[6] S. Goswami, A.J. Matula, S.P. Rath, S. Hedström, S. Saha, M. Annamalai, D. Sengupta, A. Patra, S. Ghosh, H. Jani, S. Sarkar, M.R. Motapothula, C.A. Nijhuis, J. Martin, S. Goswami, V.S. Batista, T. Venkatesan, Robust resistive memory devices using solution-processable metalcoordinated azo aromatics, Nat. Mater. 16 (2017) 1216-1224. doi:10.1038/nmat5009.

[7] Y. Dong, G. Yu, M.C. McAlpine, W. Lu, C.M. Lieber, Si/a-Si core/shell nanowires as nonvolatile crossbar switches, Nano Lett. 8 (2008) 386-391. doi:10.1021/n1073224p. 
[8] A. Khiat, P. Ayliff, T. Prodromakis, High Density Crossbar Arrays with Sub- 15 nm Single Cells via Liftoff Process Only, Sci. Rep. (2016) 1-8. doi:10.1038/srep32614.

[9] A. Blug, D. Carl, H. Höfler, Inspecting rapidly moving surfaces for small defects using CNN cameras, Soc. Photo-Optical Instrum. Eng. 8791 (2013) 1-11. doi:10.1117/12.2020568.

[10] Á. Zarándy, ed., Focal-Plane Sensor Chips, Springer-Verlag New York, 2011. doi:10.1007/978$1-4419-6475-5$.

[11] J. Müller, R. Wittig, J. Müller, R. Tetzlaff, An Improved Cellular Nonlinear Network Architecture for Binary and Grayscale Image Processing, IEEE Trans. CIRCUITS Syst. EXPRESS BRIEFS. 65 (2018) 1084-1088. doi:10.1109/TCSII.2016.2621773.

[12] A.H. Jaafar, R.J. Gray, E. Verrelli, M. O’Neill, S.M. Kelly, N.T. Kemp, Reversible optical switching memristors with tunable STDP synaptic plasticity: a route to hierarchical control in artificial intelligent systems, Nanoscale. 9 (2017) 17091. doi:10.1039/C7NR06138B.

[13] A. Emboras, I. Goykhman, B. Desiatov, N. Mazurski, L. Stern, J. Shappir, U. Levy, Nanoscale plasmonic memristor with optical readout functionality, Nano Lett. 13 (2013) 6151-6155. doi:10.1021/nl403486x.

[14] C. Hoessbacher, Y. Fedoryshyn, A. Emboras, A. Melikyan, M. Kohl, D. Hillerkuss, C. Hafner, J. Leuthold, The plasmonic memristor: a latching optical switch, Optica. 1 (2014) 198. doi:10.1364/OPTICA.1.000198.

[15] H. Tan, G. Liu, X. Zhu, H. Yang, B. Chen, X. Chen, J. Shang, W.D. Lu, Y. Wu, R.W. Li, An optoelectronic resistive switching memory with integrated demodulating and arithmetic functions, Adv. Mater. 27 (2015) 2797-2803. doi:10.1002/adma.201500039.

[16] W. Wang, G.N. Panin, X. Fu, L. Zhang, P. Ilanchezhiyan, V.O. Pelenovich, D. Fu, T.W. Kang, MoS2 memristor with photoresistive switching, Sci. Rep. 6 (2016) 31224. doi:10.1038/srep33107.

[17] M. Ungureanu, R. Zazpe, F. Golmar, P. Stoliar, R. Llopis, F. Casanova, L.E. Hueso, A lightcontrolled resistive switching memory, Adv. Mater. 24 (2012) 2496-2500. doi:10.1002/adma.201200382.

[18] K.M. Kossifos, M.A. Antoniades, J. Georgiou, A.H. Jaafar, N.T. Kemp, An Optically- 
Programmable Absorbing Metasurface, IEEE Int. Symp. Circuits Syst. (2018). doi:10.1109/ISCAS.2018.8351874.

[19] J. Georgiou, K.M. Kossifos, M.A. Antoniades, A.H. Jaafar, N.T. Kemp, Chua MemComponents for Adaptive RF Metamaterials, IEEE Int. Symp. Circuits Syst. (2018). doi:10.1109/ISCAS.2018.8351852.

[20] V.K. Nagareddy, M.D. Barnes, F. Zipoli, K.T. Lai, A.M. Alexeev, M.F. Craciun, C.D. Wright, Multilevel Ultrafast Flexible Nanoscale Nonvolatile Hybrid Graphene Oxide-Titanium Oxide Memories, ACS Nano. 11 (2017) 3010-3021. doi:10.1021/acsnano.6b08668.

[21] S. Ki Hong, J. Eun Kim, S.O. Kim, B. Jin Cho, Analysis on switching mechanism of graphene oxide resistive memory device, J. Appl. Phys. 110 (2011) 0-5. doi:10.1063/1.3624947.

[22] G. Liu, X. Zhuang, Y. Chen, B. Zhang, J. Zhu, C.X. Zhu, K.G. Neoh, E.T. Kang, Bistable electrical switching and electronic memory effect in a solution-processable graphene oxidedonor polymer complex, Appl. Phys. Lett. 95 (2009) 2007-2010. doi:10.1063/1.3276556.

[23] M. Yi, L. Zhao, Q. Fan, X. Xia, W. Ai, L. Xie, X. Liu, N. Shi, W. Wang, Y. Wang, W. Huang, Electrical characteristics and carrier transport mechanisms of write-once-read-many-times memory elements based on graphene oxide diodes, J. Appl. Phys. 110 (2011). doi:10.1063/1.3639287.

[24] A. Thakre, H. Borkar, B.P. Singh, A. Kumar, Electroforming free high resistance resistive switching of graphene oxide modified polar-PVDF, RSC Adv. 5 (2015) 57406-57413. doi:10.1039/c5ra08663a.

[25] J. Liu, Z. Yin, X. Cao, F. Zhao, L. Wang, W. Huang, H. Zhang, Fabrication of flexible, allreduced graphene oxide non-volatile memory devices, Adv. Mater. 25 (2013) 233-238. doi:10.1002/adma.201203349.

[26] D.I. Son, T.W. Kim, J.H. Shim, J.H. Jung, D.U. Lee, J.M. Lee, W. Il Park, W.K. Choi, Flexible organic bistable devices based on graphene embedded in an insulating poly(methyl methacrylate) polymer layer, Nano Lett. 10 (2010) 2441-2447. doi:10.1021/nl1006036.

[27] C.L. He, F. Zhuge, X.F. Zhou, M. Li, G.C. Zhou, Y.W. Liu, J.Z. Wang, B. Chen, W.J. Su, Z.P. Liu, Y.H. Wu, P. Cui, R.W. Li, Nonvolatile resistive switching in graphene oxide thin films, 
Appl. Phys. Lett. 95 (2009). doi:10.1063/1.3271177.

[28] S.K. Kim, J.Y. Kim, B.C. Jang, M.S. Cho, S.Y. Choi, J.Y. Lee, H.Y. Jeong, Conductive Graphitic Channel in Graphene Oxide-Based Memristive Devices, Adv. Funct. Mater. 26 (2016) 7406-7414. doi:10.1002/adfm.201602748.

[29] X. Sun, Z. Liu, K. Welsher, J.T. Robinson, A. Goodwin, S. Zaric, H. Dai, Nano-graphene oxide for cellular imaging and drug delivery, Nano Res. 1 (2008) 203-212. doi:10.1007/s12274-0088021-8.

[30] G. Eda, Y.Y. Lin, C. Mattevi, H. Yamaguchi, H.A. Chen, I.S. Chen, C.W. Chen, M. Chhowalla, Blue photoluminescence from chemically derived graphene oxide, Adv. Mater. 22 (2010) 505509. doi:10.1002/adma.200901996.

[31] K.P. Loh, Q. Bao, G. Eda, M. Chhowalla, Graphene oxide as a chemically tunable platform for optical applications, Nat. Chem. 2 (2010) 1015-1024. doi:10.1038/nchem.907.

[32] E. Kymakis, K. Savva, M.M. Stylianakis, C. Fotakis, E. Stratakis, Flexible organic photovoltaic cells with in situ nonthermal photoreduction of spin-coated graphene oxide electrodes, Adv. Funct. Mater. 23 (2013) 2742-2749. doi:10.1002/adfm.201202713.

[33] S.H. Park, H.S. Kim, Environmentally benign and facile reduction of graphene oxide by flash light irradiation, Nanotechnology. 26 (2015) 205601. doi:10.1088/0957-4484/26/20/205601.

[34] S.K. Chang-Jian, J.R. Ho, J.W. John Cheng, Y.P. Hsieh, Characterizations of photoconductivity of graphene oxide thin films, AIP Adv. 2 (2012). doi:10.1063/1.3702871.

[35] S. Chakrabarty, K. Chakraborty, A. Laha, T. Pal, S. Ghosh, Photocurrent generation and conductivity relaxation in reduced graphene oxide $\mathrm{Cd} 0.75 \mathrm{Zn} 0.25 \mathrm{~S}$ nanocomposite and its photocatalytic activity, J. Phys. Chem. C. 118 (2014) 28283-28290. doi:10.1021/jp509575p.

[36] F. Chiu, A Review on Conduction Mechanisms in Dielectric Films, Adv. Mater. Sci. Eng. ID 578168 (2014) 18. doi:10.1155/2014/578168.

[37] W. Gao, L.B. Alemany, L.J. Ci, P.M. Ajayan, New insights into the structure and reduction of graphite oxide, Nat. Chem. 1 (2009) 403-408.

[38] P. Sungjin, J. An, R.D. Piner, I. Jung, D. Yang, A. Velamakanni, S.T. Nguyen, R.S. Ruoff, 
Aqueous suspension and characterization of chemically modified graphene sheets, Chem. Mater. 20 (2008) 6592-6594.

[39] D. Dreyer, S. Park, C. Bielawski, R. Ruoff, The chemistry of graphene oxide, Chem Soc Rev. 39 (2010) 228-240.

[40] Y. Shen, S. Yang, P. Zhou, Q. Sun, P. Wang, L. Wan, J. Li, L. Chen, X. Wang, S. Ding, D.W. Zhang, Evolution of the band-gap and optical properties of graphene oxide with controllable reduction level, Carbon N. Y. 62 (2013) 157-164.

[41] A. Hunt, E.Z. Kurmaev, A. Moewes, Band gap engineering of graphene oxide by chemical modification, Carbon N. Y. 75 (2014) $366-371$.

[42] V. Berry, Impermeability of graphene and its applications, Carbon N. Y. 62 (2013) 1-10.

[43] A.B. Kaiser, C. Gómez-Navarro, R.S. Sundaram, M. Burghard, K. Kern, Electrical Conduction Mechanism in Chemically Derived Graphene Monolayers, Nano Lett. 9 (2009) 1787-1792. doi:10.1021/n1803698b.

[44] H.Y. Jeong, J.Y. Kim, J.W. Kim, J.O. Hwang, J.E. Kim, J.Y. Lee, T.H. Yoon, B.J. Cho, S.O. Kim, R.S. Ruoff, S.Y. Choi, Graphene oxide thin films for flexible nonvolatile memory applications, Nano Lett. 10 (2010) 4381-4386. doi:10.1021/nl101902k.

[45] S.K. Kim, J.Y. Kim, S.Y. Choi, J.Y. Lee, H.Y. Jeong, Direct Observation of Conducting Nanofilaments in Graphene-Oxide-Resistive Switching Memory, Adv. Funct. Mater. 25 (2015) 6710-6715. doi:10.1002/adfm.201502734.

[46] T. Ohno, T. Hasegawa, T. Tsuruoka, K. Terabe, J.K. Gimzewski, Short-term plasticity and longterm potentiation mimicked in single inorganic synapses, Nat. Mater. 10 (2011) 591-595. doi:10.1038/nmat3054.

[47] H. Xia, Q. Chen, H.-B. Sun, L. Guo, S. Wei, F.-S. Xiao, Y. Zhang, Y. He, Direct imprinting of microcircuits on graphene oxides film by femtosecond laser reduction, Nano Today. 5 (2010) 15-20. doi:10.1016/j.nantod.2009.12.009.

[48] M.F. El-Kady, V. Strong, S. Dubin, R.B. Kaner, Laser scribing of high-performance and flexible graphene-based electrochemical capacitors, Science (80-. ). 335 (2012) 1326-1330. doi:10.1126/science. 1216744 . 
[49] X. Qi, X. Zou, Z. Huang, L. Ren, G. Hao, Y. Liu, Ultraviolet, visible, and near infrared photoresponse properties of solution processed graphene oxide, Appl. Surf. Sci. 266 (2013) 332336.

[50] B. Chitara, S.B. Krupanidhi, C.N.R. Rao, Solution processed reduced graphene oxide ultraviolet detector, Appl. Phys. Lett. 99 (2011) 1-4. doi:10.1063/1.3640222.

[51] S.M. Mok, F. Yan, H.L.W. Chan, S.M. Mok, F. Yan, H.L.W. Chan, Organic phototransistor based on poly (3-hexylthiophene)/Ti O 2 nanoparticle composite, Appl. Phys. Lett. 023310 (2013). doi:10.1063/1.2957981. 